\title{
QUALIDADE EM SERVIÇOS PÚBLICOS DE SAÚDE: UMA AVALIAÇÃO DA ESTRATÉGIA SAÚDE DA FAMÍLIA
}

\section{QUALITY IN PUBLIC HEALTH SERVICES - AN ASSESSMENT OF THE FAMILY HEALTH STRATEGY}

\author{
Angela Weber Righi angelawrighi@yahoo.com.br \\ Alberto Souza Schmidt* albertoschmidt56@gmail.com \\ Jonas Cardona Venturini" ${ }^{\star \star}$ jonasventurini@yahoo.com.br \\ * Universidade Federal de Santa Maria (UFSM) \\ ** Universidade Federal do Rio Grande do Sul (UFRGS)
}

\begin{abstract}
Resumo: Esta pesquisa objetivou avaliar a qualidade da Estratégia Saúde da Família (ESF) através da mensuração da satisfação dos usuários em relação aos serviços prestados, identificando a relação entre esta satisfação e a percepção da qualidade pelos funcionários. Sendo assim, caracterizou-se como um estudo de caso, descritivo e quantitativo, desenvolvido junto a 606 usuários e 39 profissionais pertencentes a cinco unidades da ESF em um município da Região Central do Rio Grande do Sul, através de questionários baseados na Escala SERVQUAL. Como resultado obteve-se uma avaliação negativa da ESF por ambos os atores: usuários mostram-se insatisfeitos e profissionais caracterizam como inaceitável a qualidade dos serviços. Torna-se importante destacar que nenhum ponto foi avaliado como satisfatório ou de qualidade excelente. Este estudo contribuiu para análise e reorganização das práticas utilizadas atualmente pela ESF na prestação de seus serviços, tendo os aspectos aqui identificados como norteadores de ações de melhoria.
\end{abstract}

Palavras-chave: Avaliação da qualidade em serviços. Escala SERVQUAL. Saúde pública. Estratégia Saúde da Família.

\begin{abstract}
This study aimed to evaluate the quality of the Family Health Strategy (ESF) through the measurement of users' satisfaction regarding services provided, identifying the relationship between the satisfaction and perception of quality by the officials. Therefore, it is characterized as a case study, descriptive and quantitative, developed with 606 users and 39 professionals belonging to five units of the ESF in a municipality of the Central Region of Rio Grande do Sul, through questionnaires based on the SERVQUAL Scale. The result obtained is a negative evaluation of the ESF by both actors, users show a dissatisfied and professionals characterized as unacceptable the quality of services. It is important to emphasize that no point was assessed as satisfactory or excellent quality. This study contributed to analysis and reorganization of the practices currently used by the ESF in the provision of its services, and the issues identified here as guiding the actions of improvement.
\end{abstract}

Keywords: Evaluation of quality. SERVQUAL Scale. Public health. Family Health Strategy.

\section{INTRODUÇÃO}

O desenvolvimento da gestão da qualidade ao longo do tempo fez este movimento de gestão transpor o seu domínio industrial e adquirir proeminência em 
todos os setores de atividade, fazendo parte, cada vez mais, do conjunto de idéias de base que norteiam a administração pública e a vida dos cidadãos. O cenário mundial evidência que a qualidade não pode ser mais considerada como opcional aos serviços e sim requisito fundamental para sobrevivência. E, mais importante do que isso, é uma responsabilidade social e ética.

A característica de responsabilidade ética e social da qualidade em serviços torna-se ainda mais importante quando direcionada aos serviços públicos. As organizações do setor público são as maiores prestadoras de bens e serviços, tendo como principal característica uma relação de responsabilidade direta com a sociedade (ESTEFANO, 1996). A qualidade tem se inserido nessas organizações, porém de forma ainda incipiente se comparada com outros setores. Munro (1994 apud FADEL; FILHO, 2006) afirma que a prestação de serviços realizada pelo poder público mantém em foco a própria existência do serviço, deixando sua qualidade relegada ao segundo plano.

Os serviços de saúde não se furtam dessa realidade. Ao longo dos anos, a qualidade na saúde foi estabelecida por aqueles que providenciavam o serviço, os profissionais da saúde. Somente na década de 80 é que o setor da saúde voltou-se para a qualidade de uma forma mais gerencial e ativa, como já vinha ocorrendo no campo industrial. Esse cenário é ainda mais recente nos serviços públicos de saúde.

Analisando-se de forma breve o histórico do sistema de saúde no Brasil, identificam-se como características a centralização federal; desigualdade de acesso; divisão entre ações de prevenção e reabilitação; utilização irracional dos recursos humanos, tecnológicos e financeiros; e, pela prática dissociada do contexto de vida dos sujeitos; acarretando baixa resolutividade dos problemas de saúde e gerando alto grau de insatisfação, tanto na população, como nos gestores e profissionais da saúde (BARROS, 1997; BRASIL, 2000a; SOUZA, 2000).

Numa tentativa de reorganizar o sistema e propor novas formas de atuação da saúde pública, visando maior acesso e qualidade aos serviços, o Governo Federal lançou em 1994 o Programa de Saúde da Família (PSF), que propôs mudanças na forma de cuidar da saúde, principalmente em relação ao objeto da atenção e à maneira de organização do serviço e das ações: o foco passa a ser o indivíduo, a família e seu ambiente físico e social, com a atenção à saúde orientada 
pelo princípio da vigilância à saúde, combinando ações de promoção, prevenção e cura, desenvolvidas por uma equipe multidisciplinar de profissionais que têm responsabilidade sanitária sobre um território definido (BRASIL, 2008).

A despeito de ter iniciado como um programa, o PSF encontra-se hoje na condição de estratégia prioritária para a organização da atenção básica, "de acordo com os preceitos do Sistema Único de Saúde" (BRASIL, 2006a, p. 11). Sendo assim, muda sua denominação de Programa de Saúde da Família para Estratégia Saúde da Família - ESF (BRASIL, 2009). Sendo a ESF estratégia prioritária do Governo Federal, fica evidente que a adoção dos princípios da qualidade torna-se fundamental para que os objetivos propostos sejam atingidos. Dessa forma, iniciativas de avaliação dos programas desenvolvidos mostram-se como uma alternativa para o início de uma gestão pela qualidade nos serviços públicos de saúde.

A utilização da avaliação como etapa inicial num processo de busca pela qualidade está embasada em um consenso, evidenciado por pesquisadores e gestores das mais diferentes áreas de atuação, sobre a relevância da avaliação de serviços para a gestão pela qualidade, pois esta favorece diretrizes e opções para o processo de planejamento e possibilita um controle técnico e social dos serviços e programas prestados a sociedade. No campo da saúde pública esta avaliação toma proporções ainda mais significativas, devido a complexidade que envolve este setor de atividade (HARTZ; POUVOURVILLE, 1998).

O processo de avaliação da qualidade em serviços de saúde envolve tanto quem utiliza os serviços como quem os produz. Não há dúvida de que usuário e prestador ocupam posições diferentes no processo, embora ambos contribuam para que os serviços sejam executados. Assim, as expectativas e necessidades de usuários e prestadores determinam de maneira diversa a qualidade dos serviços. Sendo assim, torna-se essencial entender como estes principais atores percebem a qualidade dos serviços que estão sendo prestados, visando identificar e analisar as diferentes percepções para, posteriormente, intervir buscando maneiras de alinhálas de modo a satisfazer as necessidades de ambos e promover a melhoria contínua. 
Cabe ressaltar que, a avaliação surge como um importante instrumento de mudança, que não deve ser visto como uma ameaça, mas como um incentivo para que os serviços de saúde cumpram padrões mínimos de qualidade e promovam uma renovação da sua cultura de trabalho.

De acordo com o exposto, que evidencia a importância da ESF para a saúde pública brasileira e a necessidade da utilização da gestão pela qualidade para melhoria dos serviços prestados por este setor, o presente estudo tem como objetivo avaliar a qualidade da Estratégia Saúde da Família (ESF) através da mensuração da satisfação dos usuários em relação aos serviços prestados, buscando identificar a relação existente entre esta satisfação e a percepção da qualidade dos serviços pelos funcionários.

\section{AVALIAÇÃO DA QUALIDADE EM SERVIÇOS DE SAÚDE}

Ao pesquisar-se sobre as teorias que norteiam a qualidade em saúde encontra-se uma gama de conceitos e de perspectivas diferentes sobre o que é essa qualidade, às vezes divergentes e, em alguns casos, antagônicos (SOUZA JúNIOR, 2002). Esta diversidade torna as questões relacionadas com a qualidade na prestação de serviços de saúde difíceis e complexas de abordar.

Mezomo (2001), ao compartilhar desta idéia, a complementa dizendo que a qualidade se torna mais complexa na área da saúde porque não age em função de um único serviço (produto final), pois este é sempre incerto, não obedece a padrões estabelecidos e não tem qualidade uniforme.

Dessa forma, as peculiaridades dos serviços de saúde devem ser observadas ao buscar a qualidade na prestação dos mesmos. Apoiado nos conceitos de Garvin (1990), Urdan (2001) cita três singularidades principais das instituições de saúde: não há uma clareza na conexão entre entradas e saídas; os pacientes geralmente têm dificuldades em avaliar aspectos técnicos; e existe, em grandes hospitais, duas linhas de autoridades distintas, o administrativo e o médico.

Avedis Donabedian, um dos principais estudiosos sobre a qualidade em saúde, tem seus conceitos utilizados até hoje pelas organizações de saúde, principalmente nos aspectos referentes a avaliação da qualidade em serviços de 
saúde (CAMPOS; CARVALHO, 2000; VUORI, 1991). Para Donabedian (1980), uma definição para qualidade deveria se iniciar a partir de três dimensões: a estrutura, o processo e o resultado. A estrutura envolve os recursos físicos, humanos, materiais, equipamentos e financeiros necessários para a assistência médica. O processo, que se refere às atividades envolvendo os profissionais da saúde e usuários, inclui o diagnóstico; o tratamento; e, os aspectos éticos da relação médico, profissional, equipe de saúde e paciente. O resultado corresponde ao produto final da assistência prestada, considerando a saúde, satisfação dos padrões e expectativas dos usuários.

Anos mais tarde, Donabedian (1990) ampliou esses princípios, utilizando-se do denominado "Sete Pilares da Qualidade": eficácia, efetividade, eficiência, otimização, aceitabilidade, legitimidade, equidade, descritos no quadro 1.

\begin{tabular}{|c|c|}
\hline DIMENSÕES & DESCRIÇȦO DA DIMENSÃO DA QUALIDADE EM SAÚDE \\
\hline EFICÁCIA & $\begin{array}{l}\text { É a capacidade do cuidado, na sua forma mais perfeita, de contribuir para } \\
\text { a melhoria das condições de saúde, ou seja, capacidade de a arte e a } \\
\text { ciência da saúde produzirem melhorias na saúde e no bem-estar. Significa } \\
\text { o melhor que se pode fazer nas condições mais favoráveis, dado o estado } \\
\text { do paciente e mantidas constantes as demais circunstâncias. }\end{array}$ \\
\hline EFETIVIDADE & $\begin{array}{l}\text { É o quadro de melhorias possíveis nas condições de saúde obtido. } \\
\text { Melhoria na saúde, alcançada ou alcançável nas condições usuais da } \\
\text { prática cotidiana. Ao definir e avaliar a qualidade, a efetividade pode ser } \\
\text { mais precisamente especificada como sendo o grau em que o cuidado, } \\
\text { cuja a qualidade está sendo avaliada, alça-se ao nível de melhoria da } \\
\text { saúde que os estudos de eficácia têm estabelecido como alcançáveis. }\end{array}$ \\
\hline EFICIÊNCIA & $\begin{array}{l}\text { É a medida do custo com o qual uma dada melhoria na saúde é } \\
\text { alcançada. Se duas estratégias de cuidado são igualmente eficazes e } \\
\text { efetivas, a mais eficiente é a de menor custo. }\end{array}$ \\
\hline OTIMIZAÇÃO & $\begin{array}{l}\text { Torna-se relevante à medida que os efeitos do cuidado da saúde não são } \\
\text { avaliados de forma absoluta, mas relativamente aos custos. Numa curva } \\
\text { ideal, o processo de adicionar benefícios pode ser tão desproporcional } \\
\text { aos custos acrescidos, que tais "adições" úteis perdem a razão de ser. }\end{array}$ \\
\hline DIMENSÕES & DESCRIÇȦO DA DIMENSÃO DA QUALIDADE EM SAÚDE \\
\hline ACEITABILIDADE & $\begin{array}{l}\text { Sinônimo de adaptação do cuidado aos desejos, expectativas e valores } \\
\text { dos pacientes e suas famílias. Depende da efetividade, eficiência e } \\
\text { otimização, além da acessibilidade ao cuidado, das características da } \\
\text { relação médico-paciente e das amenidades do cuidado, aos efeitos e ao } \\
\text { custo do serviço prestado. }\end{array}$ \\
\hline LEGITIMIDADE & $\begin{array}{l}\text { Aceitabilidade do cuidado da forma em que é visto pela comunidade ou } \\
\text { sociedade em geral. É a conformidade com as preferências sociais. }\end{array}$ \\
\hline EQUIDADE & $\begin{array}{l}\text { Princípio pelo qual se determina o que é justo ou razoável na distribuição } \\
\text { do cuidado e de seus benefícios entre os membros da população. A } \\
\text { equidade é parte daquilo que torna o cuidado aceitável para os indivíduos } \\
\text { e legítimo para a sociedade. Igualdade na distribuição do cuidado e de } \\
\text { seus efeitos sobre a saúde. }\end{array}$ \\
\hline
\end{tabular}

Quadro 1: Os Sete Pilares da Qualidade

Fonte: Adaptado de Donabedian (1994) 
Particularmente para este estudo, a satisfação do usuário como fator que determina a qualidade é de considerável relevância. Nesta direção, Tanaka e Melo (2004) afirmam que ao realizar a avaliação em sistemas e/ou serviços de saúde deve-se ter em mente que os serviços prestados têm como finalidade os usuários, que são parte do processo de organização e prestação destas ações.

A importância da ótica dos usuários quanto aos serviços prestados é evidenciada também por Gemmel et al. (2002). Estes autores transferiram os conceitos desenvolvidos por Gronroos (2003) das dimensões técnicas e funcional na qualidade em serviços para o setor de saúde. Eles propuseram que a qualidade em saúde é formada por duas dimensões: a qualidade técnica, que corresponde a exatidão do diagnóstico e de procedimentos; e a qualidade funcional, que se refere à maneira com que os serviços são entregues aos pacientes.

Segundo Urdan (2001), os pacientes quase sempre conhecem pouco sobre os aspectos de qualidade técnica, mas, em geral, não têm dificuldade em avaliar o lado interpessoal. Outros autores compartilham dessa mesma opinião, acreditando ser essa a base do julgamento na qualidade de um serviço de saúde por parte dos usuários (DONABEDIAN, 1990; GEMMEL et al., 2002).

A boa relação entre os profissionais e o paciente é a maior contribuição que se pode oferecer ao usuário, com efeitos positivos no tratamento. Em alguns casos, este fator determina diferenças sensíveis entre dois serviços iguais. Acredita-se que essa "boa relação" pode compensar falhas do cuidado técnico.

Incentivos a humanização da assistência a saúde estão ocorrendo de forma progressiva, além da incorporação de noções relacionadas à cidadania, aos direitos do consumidor e à responsabilidade ética dos profissionais (NOGUEIRA-MARTINS, 2003). A qualidade de um serviço de saúde está diretamente relacionada à qualidade da relação interpessoal que ocorre entre os pacientes e os profissionais encarregados da assistência à saúde.

Em uma pesquisa sobre qualidade em serviços de saúde realizada no município de Recife - PE - BR, Assunção (2006) identificou que, em uma escala que variava de (1) péssima a (5) ótima, usuários com nível de instrução até o ensino fundamental consideram boa a qualidade típica dos serviços privados. Já para pessoas que estão cursando ou já concluíram o nível superior ou curso técnico, a 
qualidade dos serviços de saúde privado do país está entre razoável e boa. Nota-se que, na medida em que o grau de instrução aumenta, a valoração da qualidade diminui.

Quando questionados sobre a qualidade do serviço público de saúde, a situação é semelhante. Novamente a valoração da qualidade cai conforme aumenta o nível de instrução dos usuários, sendo que a condição de saúde do setor público apresenta, em média, qualidade ruim (ASSUNÇÃO, 2006).

Assunção (2006) afirma ainda que as longas filas, as condições precárias de atendimento e o tratamento desumano da população, frequentemente divulgado pela mídia, corroboram para denegrir cada vez mais a imagem das instituições públicas de saúde.

Outros dados pertinentes à qualidade em serviços de saúde surgem na pesquisa realizada por Blendon et al. (2001). A pesquisa realizada com médicos da Austrália, do Canadá, dos Estados Unidos, da Grã-Bretanha e da Nova Zelândia identificou que a maioria dos mesmos foi a favor de reformas nos sistemas de saúde para que tenham mais tempo com seus pacientes, visando um aprimoramento da qualidade. Porém, apenas $33 \%$ a $40 \%$ dos entrevistados concordavam que as avaliações dos pacientes poderiam melhorar a qualidade. Pode-se concluir, a partir disto, que esses profissionais apresentam pouca disposição em absorver o retorno dos pacientes, apesar do interesse em poder lhes dedicar mais tempo.

Em função disto, torna-se relevante identificar as duas óticas em relação à qualidade dos serviços prestados: a dos usuários e a dos profissionais. Muitas vezes os profissionais acreditam estar realizando um bom trabalho quando, na verdade, não estão atingindo as expectativas dos usuários. Ou então, a situação pode apresentar-se de modo inverso.

Parente (2000) afirma que, à medida que são apresentados melhores serviços, os clientes elevam suas expectativas. Dessa forma, a melhoria dos serviços prestados acarreta um aumento no nível de exigências e das expectativas. Em conseqüência, o aumento das expectativas estimula os prestadores de serviços a prosseguirem em seus esforços para a melhoria contínua.

Considerando estes aspectos, o presente estudo vem a contribuir com a qualidade de serviços de saúde, identificando aspectos relevantes junto aos serviços 
para a organização e planejamento das ações da saúde. Em especial, os serviços públicos de saúde, com a avaliação da qualidade da Estratégia Saúde da Família através da mensuração da satisfação dos usuários e a relação desta com a qualidade percebida dos serviços pelos profissionais atuantes no setor.

\section{SAÚDE NO BRASIL: A ESTRATÉGIA SAÚDE DA FAMÍLIA}

A década de 80 foi a época na qual se iniciaram as mais importantes mudanças no sistema público de saúde brasileiro, caracterizado por um modelo médico-assistencial privatista e excludente. Buscava-se um modelo de saúde que refletisse um sistema universal, integral e descentralizado.

As mudanças tiveram início com o movimento sanitário, que entra os anos 80 propondo como linha tática a ocupação dos espaços institucionais e a formulação/ implementação ativa de políticas de saúde. Em março de 1986, devido a um movimento sanitário mobilizado e articulado e as condições históricas do momento,

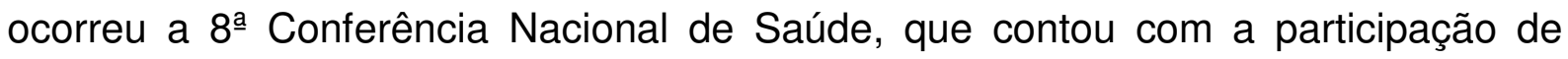
diversos setores organizados da sociedade, surgindo um consenso de que para 0 setor da saúde no Brasil não era suficiente uma mera reforma administrativa e financeira, mas sim uma mudança em todo o arcabouço jurídico-institucional vigente, que contemplasse a ampliação do conceito de saúde segundo os preceitos da reforma sanitária (ANDRADES; PONTES; MARTINS JÚNIOR, 2000).

O relatório produzido nessa Conferência serviu de referência para os constituintes que elaboraram a Constituição de 1988 e para a aprovação das Leis 8080 e 8142, em 1990, que reconhecem o direito à saúde como direito de cidadania para todos, culminando na criação do Sistema Único de Saúde (SUS).

Atualmente, cerca de $60 \%$ da população brasileira é dependente SUS, que objetiva alterar a situação de desigualdade na assistência à saúde da população, tornando obrigatório o atendimento público a qualquer cidadão, ofertando serviços com qualidade adequados às necessidades da população sem forma alguma de remuneração direta do usuário do serviço. Fazem parte desse Sistema os centros e postos de saúde, hospitais, laboratórios, hemocentros, além de fundações e institutos de pesquisa (BRASIL, 1990). 
Porém, enquanto a década de 80 foi marcada como um período de mudanças importantes no sistema de saúde brasileiro, os anos 90 ficam registrados como um período de grande crise econômica e fiscal do país, sendo a saúde afetada por esta realidade. Esse cenário existente na década de 90 levou o Governo a implantar um conjunto de programas, ações regulatórias e novas modalidades de alocação de recursos, com o objetivo de melhorar a equidade e eficácia no SUS (REIS; HORTALE, 2004). É nesse contexto, de crise econômica, constrangimentos sociais e ajustes macroeconômicos, que é implantado o Programa de Saúde da Família (PSF), em 1994.

A Estratégia Saúde de Família (denominação dada ao PSF a partir de 2003), concebida pelo Ministério da Saúde, foi destinada a contribuir para a organização de sistemas locais e reordenar o modelo assistencial a partir do modelo da Atenção Básica à Saúde. A Estratégia Saúde da Família (ESF) propõe a integração e organização das ações de saúde em um território definido, cuja atenção está centrada na família a partir do seu ambiente físico e social, possibilitando às equipes uma compreensão ampliada do processo saúde-doença e da necessidade de intervenções que vão além de práticas curativas.

A Unidade de Saúde da Família atua com princípios de integralidade e hierarquização, territorialização e adstrição da clientela, e de uma equipe multiprofissional, sendo estruturada em uma lógica de atenção à saúde, com a geração de práticas de cuidado, considerando a indissociabilidade entre os trabalhos clínicos e a promoção da saúde (BRASIL, 2000b).

De acordo com os dados do Departamento de Atenção Básica (DAB), estrutura vinculada à Secretaria de Atenção à Saúde do Ministério da Saúde, a Saúde da Família apresentou até agosto de 2005, uma adesão de 4.900 municípios, atingindo um total de 23.900 equipes implantadas e com uma cobertura populacional de $43,4 \%$ da população brasileira, o que corresponde a 76,8 milhões de pessoas (BRASIL, 2008). Dados mais recentes indicam que no período de 1994 a agosto de 2006, houve uma evolução de $84,2 \%$ da cobertura populacional brasileira, com 26.259 Equipes de Saúde da Família implantadas, abrangendo um total de 5.093 municípios (BRASIL, 2008). 
Em face de constante expansão no País da Saúde da Família, faz-se necessário desenvolver metodologias de avaliação que possam ser utilizadas no planejamento das políticas de saúde locais. Além disso, a Estratégia Saúde da Família está em curso no país a mais de uma década, e tem sido objeto de avaliação pelo próprio Ministério da Saúde, que vem indicando a necessidade de estudos locais para analisar a relação dos serviços com seus usuários.

Segundo Medina e Aquino (2002), a avaliação na Saúde da Família constitui hoje tarefa primordial para todos envolvidos no debate sobre as perspectivas de sua consolidação, e dos avanços possíveis do SUS no País.

Cabe ressaltar, nesse sentido, a importância de se dar voz aos usuários, expressando expectativas e satisfações quanto ao uso dos serviços. Em especial, na Saúde da Família, essa atitude se apresenta como fornecedora de alternativas para o processo de tomada de decisão, na direção de serviços que se voltem a atender as necessidades dos usuários e que assim possam favorecer a aceitabilidade e credibilidade destes em relação aos serviços de saúde.

Diante do exposto, reforça-se que a avaliação da satisfação do usuário é um parâmetro que representa respostas quanto suas esperanças e desejos, podendo se tornar um instrumento útil da assistência e melhoria da qualidade de vida. Juntamente com a percepção da qualidade dos serviços por parte dos profissionais, esses dados tornam-se indicadores da atual situação da ESF e norteadores das ações futuras.

\section{MÉTODO DO ESTUDO}

O presente trabalho, ao avaliar a qualidade dos serviços prestados pela Estratégia Saúde da Família em um município na Região Central do Estado do Rio Grande do Sul sob a ótica dos usuários e funcionários, utilizou do método de estudo de caso, caracterizando-se, segundo Hair et al. (2005), como uma pesquisa descritiva, de corte transversal e abordagem quantitativa.

O cenário em estudo, a ESF em um município da Região Central do Estado do Rio Grande do Sul, apresenta dezesseis Equipes de Saúde da Família divididas em quatorze Unidades de Saúde da Família. Para a realização do estudo apenas as 
unidades que contemplassem o critério de seleção determinado: que a Equipe de Saúde da Família estivesse completa.

Segundo o critério adotado, cinco unidades participaram do estudo, totalizando uma população de 20.052 usuários e 46 funcionários. Fica evidente a inviabilidade de uma coleta de dados envolvendo toda população, principalmente no que tange aos usuários, em função do tempo determinado para a pesquisa e da necessidade de um maior número de recursos humanos e financeiros para sua realização.

Apoiado neste cenário foi selecionada uma amostra, baseada em critérios estatísticos, que representasse a população. De acordo com as características da presente pesquisa, utilizou-se a amostragem probabilística, em especial, a amostragem estratificada proporcional, pois as unidades selecionadas para o estudo apresentam dimensões distintas. Foi adotado um nível de confiança de 95\% (4\% de erro amostral).

Como resultado do processo amostral, obteve-se um número de 606 usuários da ESF e 39 profissionais pertencentes às Equipes de Saúde nas cinco unidades da ESF participantes do estudo. O número de indivíduos a serem pesquisados foi alcançado durante o desenvolvimento da pesquisa.

Como instrumento para a coleta dos dados pertinentes a pesquisa fez-se 0 uso de um questionário, composto predominantemente de questões fechadas, baseado no instrumento SERVQUAL, modelo já consolidado e utilizado em diferentes setores de atividade para mensuração da qualidade em serviços, como evidenciado na literatura referente a esta área (FITZSIMMONS; FITZSIMMONS, 2005; KARATEPE, YAPA; BABAKUS, 2005; LOVELOCK; WRIGHT, 2004; ZEITHAML; BITNER, 2003; PARASURAMAN, ZEITHAML; BERRY, 1988). Para tal, foram realizadas algumas modificações no modelo original, tendo em vista a adaptação do instrumento para a realidade em estudo. Os autores do instrumento, Parasuraman, Zeithaml e Berry (1988), evidenciam essa capacidade de adaptação do instrumento para realidades distintas.

O instrumento utilizado na pesquisa contou com uma parte inicial que objetivou conhecer o perfil dos respondentes, sendo distinto para cada um dos grupos. A segunda e terceira etapas estavam relacionadas ao instrumento 
SERVQUAL, apresentando-se compostas de 40 questões (20 expectativa e 20 percepção) contemplando as cinco dimensões da qualidade: tangibilidade, confiabilidade, responsividade, segurança e empatia. A mensuração nessas etapas deu-se através de um Escala Likert de cinco pontos, que varia de 1 (discordo totalmente) a 5 (concordo totalmente).

Por fim, a quarta parte dos questionários buscou identificar o grau de importância para cada uma das dimensões e a nota geral atribuída a ESF e aos grupos de profissionais, podendo esta nota variar de zero a dez, assim como a importância de cada dimensão.

Para atendimento dos objetivos propostos no estudo, a coleta de dados primários ocorreu através de pesquisa de campo com aplicação de questionários nos atores selecionados. Com os usuários, a coleta foi realizada mediante visita ao domicílio realizada pelo pesquisador. Estavam disponíveis os mapas das regiões de abrangência de cada Unidade e os domicílios foram escolhidos aleatoriamente. No momento da visita, era solicitado ao usuário que respondesse o questionário autoadministrado e, quando isso não era possível devido a baixa instrução do respondente, o pesquisador realizava uma entrevista estruturada para obtenção dos dados.

No que se refere à coleta dos dados primários junto aos profissionais, esta ocorreu através da entrega dos questionários aos mesmos em uma reunião para explanação dos objetivos e métodos da pesquisa.

Para apreciação e análise dos dados obtidos foram utilizados instrumentos descritivos associados a mecanismos derivados da lógica da matemática, concernentes às características deste estudo. Os questionários foram processados em microcomputadores com utilização do software SPSS, versão 13.0, para análise estatística.

A mensuração da qualidade dos serviços foi obtida da diferença entre a Percepção e a Expectativa para cada respondente. Os escores médios para cada dimensão são calculados através das respostas de todos os participantes da pesquisa em cada grupo. Escores positivos refletem um nível de satisfação elevado (serviço melhor do que o esperado); escores negativos sinalizam uma qualidade 
abaixo do esperado e, em conseqüência, respondentes insatisfeitos com o serviço; e, o escore zero implica em qualidade satisfatória.

Elucidado o método utilizado na execução da pesquisa, passa-se para a apresentação dos resultados no tópico seguinte.

\section{RESULTADOS OBTIDOS}

Este estudo foi desenvolvido com dois atores distintos, usuários e profissionais da Estratégia Saúde da Família nas unidades selecionadas, resultando, portanto, em dois construtos principais de resultados. Visando sintetizar a apresentação dos mesmos, optou-se por relatá-los evidenciando a relação existente entre as opiniões desses atores. Sendo assim, buscou-se relacionar o que cada grupo de atores identifica como mais importante e/ou mais crítico, para que ações visando a melhoria dos serviços sejam empreendidas e maiores esforços sejam dispensados a estes fatores.

De uma forma geral, os resultados obtidos mostram uma insatisfação como serviço por parte dos usuários e a atribuição de qualidade inaceitável para o serviço por parte dos profissionais. Todas as questões pesquisadas através do instrumento SERVQUAL adaptado para o estudo obtiveram Gaps negativos para ambos os atores.

Considerando as cinco dimensões da qualidade abordadas neste estudo tangibilidade, confiabilidade, responsividade, segurança e empatia - o gráfico 1 apresenta os Gaps obtidos para cauma no que tange aos usuários e funcionários.

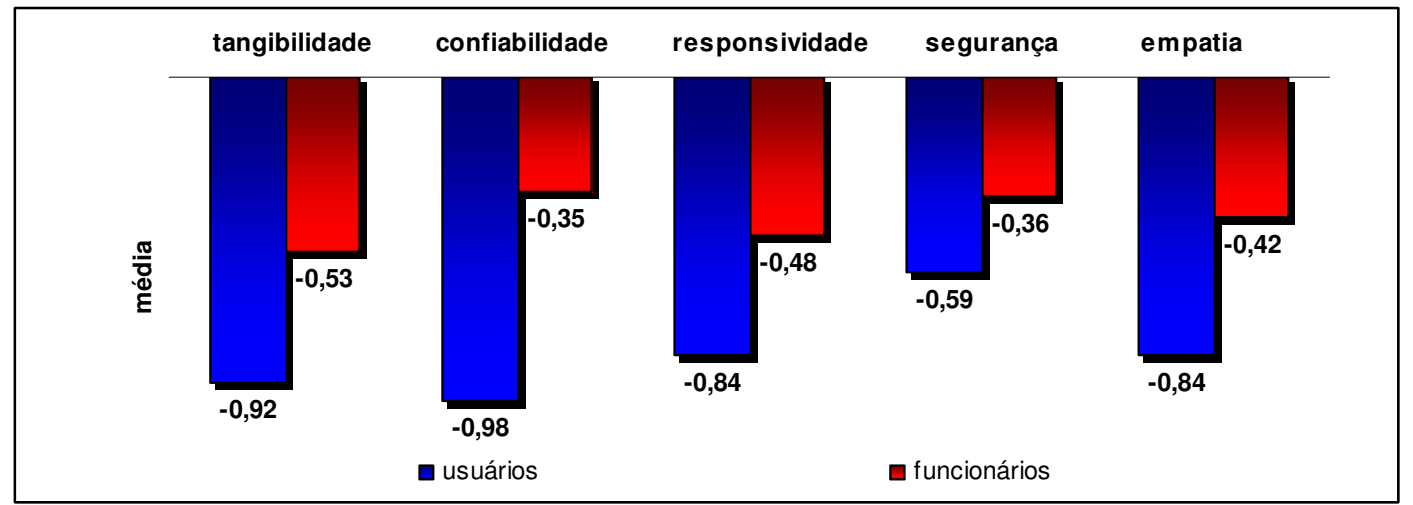

Gráfico 1: Resultados dos Gaps em cada dimensão para ambos os grupos 
A dimensão confiabilidade foi a que obteve pior avaliação por parte dos usuários, com Gap de -0,98. Já para os profissionais, essa dimensão foi a que se apresentou melhor, com Gap de -0,35. Sendo a dimensão confiabilidade relacionada a precisão dos diagnósticos; ao cumprimento de datas e horários, percebe-se a significativa insatisfação dos usuários quanto a esses quesitos, enquanto os funcionários, apesar de entenderem que esta dimensão se apresenta com qualidade inaceitável, a atribuem melhor avaliação segundo as demais.

A dimensão tangibilidade apresenta-se com a pior avaliação por parte dos funcionários (Gap= $-0,53$ ) e como segunda pior avaliação (Gap=-0,92) para os usuários. Estando essa dimensão relacionada principalmente a infra-estrutura, condições de higiene e limpeza e, sendo estes fatores primordiais em um serviço de saúde, a avaliação negativa de ambos os atores evidencia aspectos importantes a serem melhorados.

No que tange a importância atribuída a cada dimensão, o gráfico 2 mostra os valores médios obtidos em cada grupo pesquisado. Ambos os atores consideram a dimensão empatia como a mais importante ao avaliar serviços de saúde. A importância dispensada a esta dimensão, que abrange aspectos como paciência no cuidado; capacidade de recordar visitas anteriores; e tratamento mais humanizado, corrobora com a idéia de Gemmel et al (2002), de que os usuários se sentem mais capazes de avaliar os aspectos referentes a relação interpessoal determinada durante a prestação do serviço.

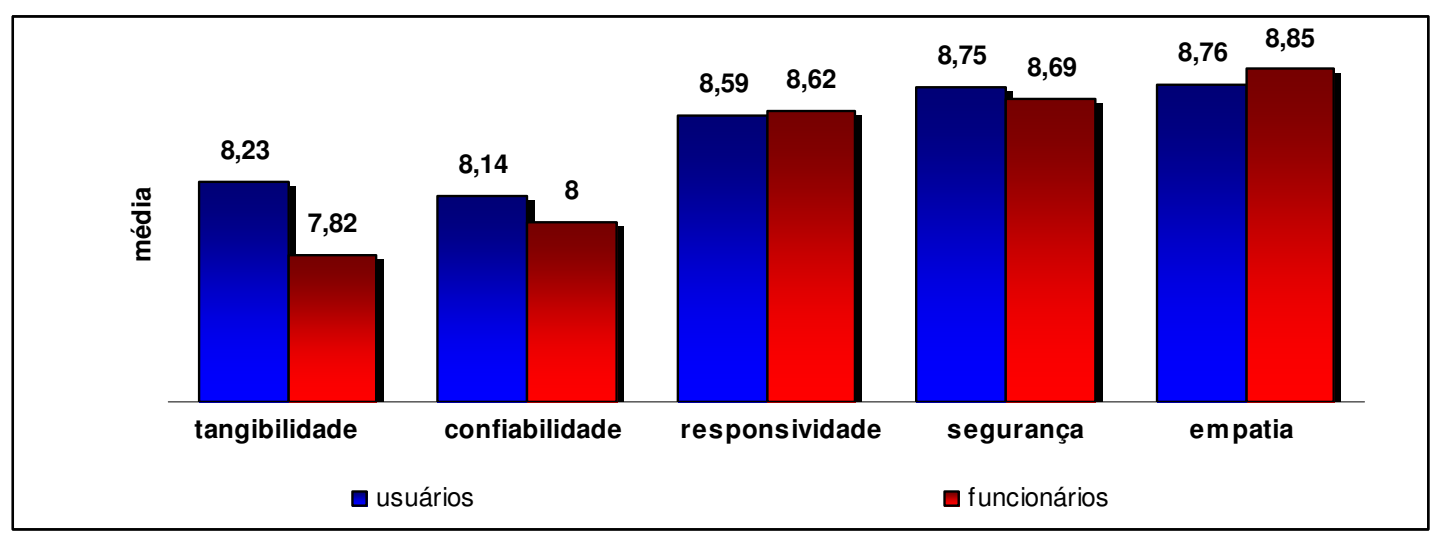

Gráfico 2: Resultados da importância de cada dimensão para ambos os grupos 
Os profissionais, ao também entenderem a dimensão empatia como mais importante, devem manter suas ações norteadas a satisfazer as necessidades referentes a estes aspectos durante a prestação de seus serviços, buscando uma maior relação interpessoal com seus pacientes.

Ainda referente a importância atribuída em cada dimensão, usuários e funcionários compartilham de mesma opinião ao determinarem como segunda mais importante a dimensão segurança, seguida da dimensão responsividade em terceiro lugar. Percebe-se que, ao avaliar serviços de saúde, usuários e profissionais consideram a importância de cada dimensão de forma semelhante. Esse fato pode auxiliar no entendimento por parte dos profissionais das necessidades dos usuários, buscando satisfazê-las para que ocorra a melhoria da qualidade dos serviços prestados.

Para cada dimensão, um ponto apresenta-se como mais crítico na avaliação de ambos os atores, expressado através da questão com maior Gap negativo dentro da dimensão. O quadro 2 mostra quais são esses pontos, juntamente com os valores de seus Gaps.

Evidencia-se que em três dimensões - responsividade, segurança e empatia os atores apresentam como item critico as mesmas questões, com os Gaps dos usuários apresentando-se sempre mais negativos. Principalmente no que tange aos funcionários, ao apresentarem como item crítico o mesmo citado pelos usuários, estes sinalizam uma consciência de que aspectos devem ser melhorados, do que o usuário espera na hora do atendimento. Estar ciente do que o usuário deseja, do que ele espera na prestação do serviço, torna mais fácil a busca da sua satisfação. 


\begin{tabular}{|c|c|c|c|c|}
\hline DIMENSÕES & USUÁRIOS & Gap & FUNCIONÁRIOS & Gap \\
\hline Tangibilidade & $\begin{array}{l}\text { O ambiente físico e as } \\
\text { instalações da unidade são } \\
\text { limpas e agradáveis }\end{array}$ & $-1,44$ & $\begin{array}{l}\text { a unidade apresenta boas } \\
\text { condições para o repouso, } \\
\text { com barulho dentro de } \\
\text { limites suportáveis }\end{array}$ & $-0,82$ \\
\hline Confiabilidade & $\begin{array}{l}\text { A unidade informa } \\
\text { precisamente a data e } \\
\text { horário do serviço a ser } \\
\text { prestado }\end{array}$ & $-1,11$ & $\begin{array}{l}\text { os funcionários da unidade } \\
\text { realizam as atividades } \\
\text { conforme o prometido }\end{array}$ & $-0,48$ \\
\hline Responsividade & $\begin{array}{l}\text { Os funcionários da unidade } \\
\text { respondem na hora as } \\
\text { solicitações dos usuários, } \\
\text { mesmo estando ocupados }\end{array}$ & $-0,93$ & $\begin{array}{l}\text { os funcionários da unidade } \\
\text { respondem na hora as } \\
\text { solicitações dos usuários, } \\
\text { mesmo estando ocupados }\end{array}$ & $-0,72$ \\
\hline Segurança & $\begin{array}{l}\text { Os funcionários utilizam } \\
\text { linguagem clara e precisa } \\
\text { ao atender seus clientes } \\
\text { a unidade é de fácil acesso } \\
\text { e bem localizada }\end{array}$ & $\begin{array}{l}-0,74 \\
-0,74\end{array}$ & $\begin{array}{l}\text { a unidade é de fácil acesso } \\
\text { e bem localizada }\end{array}$ & $-0,41$ \\
\hline Empatia & $\begin{array}{l}\text { Os funcionários da unidade } \\
\text { dão apoio moral aos } \\
\text { usuários, principalmente } \\
\text { aos mais debilitados }\end{array}$ & $-1,01$ & $\begin{array}{l}\text { os funcionários da unidade } \\
\text { dão apoio moral aos } \\
\text { usuários, principalmente } \\
\text { aos mais debilitados }\end{array}$ & $-0,64$ \\
\hline
\end{tabular}

Quadro 2: Questões com pior avaliação em cada dimensão para ambos os atores

Com relação a nota geral atribuída a ESF, os usuários se mostram mais críticos e insatisfeitos, pois suas opiniões resultaram numa nota média de 7,81. Já os profissionais indicam como nota geral para a ESF o valor de 8,21.

Referente as notas atribuídas as categorias profissionais, usuários e funcionários dedicam maior nota aos profissionais médicos. Os agentes comunitários foram os que receberam a menor nota na opinião dos usuários; enquanto para os profissionais, a categoria dos enfermeiros se apresenta com média mais baixa. Para ambas as categorias com menor nota a média foi de 7,92.

Numa análise geral, como pode ser observado pelo exposto no decorrer deste tópico, ambos os atores pesquisados, usuários e funcionários, avaliaram negativamente os serviços da ESF. Os usuários se mostram insatisfeitos e os profissionais caracterizam como inaceitável a qualidade dos serviços.

Entretanto, nota-se que os profissionais atribuíram, em todas as questões, valores superiores aos informados pelos usuários caracterizando, portanto, uma avaliação ligeiramente melhor dos funcionários. 
De qualquer forma, fica evidenciada a preocupação que devem ter gestores e coordenadores ao analisar esta pesquisa. Nenhum ponto foi avaliado como satisfatório ou de qualidade excelente pelos atores. É necessário que as práticas utilizadas atualmente pela ESF na prestação de seus serviços sejam analisadas e repensadas, utilizando os aspectos aqui identificados como norteadores de ações de melhoria.

\section{CONSIDERAÇÕES FINAIS}

A avaliação da qualidade em serviços de saúde vem se tornando, cada vez mais, um aspecto importante do processo de tomada de decisão por parte de gestores e colaboradores. Essa avaliação aumenta a probabilidade de obter-se, no final, resultados melhores do que se teria sem ela, e maior probabilidade de encontrar resultados não esperados.

Este trabalho, ao avaliar a qualidade dos serviços prestados pela Estratégia Saúde da Família (ESF) sob duas óticas distintas, usuários e profissionais, visou colaborar com esse processo de tomada de decisão por parte dos próprios profissionais, gestores e até mesmo Governo, já que se trata de um serviço público.

Foram identificados usuários insatisfeitos com os serviços e profissionais considerando de qualidade inaceitável os serviços prestados. A relação entre essas duas opiniões mostra que ambos os atores pesquisados avaliam de forma semelhante os serviços, negativamente na sua grande maioria, como pôde ser observado. Eles, apesar de ocuparem diferentes posições no processo, consideram em comum vários pontos críticos e dão importância semelhante as dimensões pesquisadas.

Dessa forma, entende-se que o trabalho realizado apresenta contribuição significativa para o setor da saúde em estudo, pois mostra um panorama da qualidade que este vem oferecendo sob a ótica de seus dois principais atores. E este panorama não se mostrou positivo, o que demonstra a necessidade de ações de melhoria.

Sabe-se que a avaliação realizada é apenas uma parte de todo um processo avaliativo maior, incluindo outras variáveis pertinentes ao estudo da área da saúde, 
que contemplem outros aspectos não evidenciados por esta pesquisa. Entretanto, seu valor se mostra significativo, pois foi pioneira nesse sentido, dando voz aos usuários e profissionais através de uma forma objetiva e mensurável, alertando para necessidades imediatas de mudanças, visto a avaliação negativa obtida.

No que tange a Engenharia de Produção, este trabalho se demonstra válido no sentido de comprovar que seus princípios e ferramentas, principalmente com relação a qualidade em serviços, podem ser utilizados no setor de serviços de saúde. O instrumento utilizado se mostrou útil para o objetivo proposto e sua aplicação tornou-se um momento importante, rico em informações que provavelmente auxiliarão na escolha de ações de melhoria.

Os limitantes deste estudo versam para a impossibilidade de se generalizar esses resultados, pelo fato de terem sido pesquisadas apenas cinco Unidades de Saúde da Família no município em estudo. Futuros trabalhos devem ser realizados, do ponto de vista teórico e empírico, na intenção de refutar ou corroborar as relações demonstradas, e identificar um panorama de todo o município, através da expansão da pesquisa para todas as Unidades de Saúde da Família.

A constante avaliação da qualidade do serviço seria de grande valia para um acompanhamento do desempenho do PSF, bem como avaliação das ações de melhoria impostas ao longo do tempo.

\section{REFERÊNCIAS}

ANDRADES, L. O. M. de; PONTES, R. J. S.; MARTINS JUNIOR, T. A descentralização no marco da Reforma Sanitária no Brasil. Revista Panamericana Salud Publica, Washington, v. 8, n. 1-2, p. 85-91, july/aug, 2000.

ASSUNÇÃO, R. S. Fatores condicionantes da qualidade do sistema de saúde na visão dos médicos e da população do Recife. 2006. 131f. Dissertação (Mestrado em Engenharia de Produção) - Universidade Federal de Pernambuco, Recife, 2006.

BRASIL. Ministério da Saúde. Departamento de atenção Básica. Diretriz conceitual da estratégia saúde da família. Disponível em:<http://dtr2004.saude.gov.br/dab/atencaobasica.php>. Acesso em: 16 fev. 2009.

. Ministério da Saúde. Departamento de atenção básica. Disponível em:<http://dtr2004.saude.gov.br/dab/docs/publicacoes/geral/avaliacao ab portugues .pdf>. Acesso em: 23 jun. 2008. 
DF, 2006a.

Ministério da Saúde. Política nacional de atenção básica. Brasília,

. Ministério da Saúde. Secretaria de Gestão Estratégica e Participativa.

A construção do SUS: histórias da reforma sanitária e do processo participativo. Brasília, DF, 2006b.

. Ministério da Saúde. Secretaria de Políticas de Saúde. Departamento de Atenção Básica. Programa de saúde da família: a implantação da unidade de saúde da família. Cadernos de Atenção Básica, caderno 1. Brasília, DF, 2000a.

. Ministério da Saúde. Secretária de Políticas Públicas. Departamento de Atenção Básica. Programa de saúde da família. Cadernos de Atenção Básica Brasília, DF, 2000b.

. Lei orgânica da saúde $\mathbf{n}^{\circ} \mathbf{8 0 8 0}$, de 19 set. 1990. Diário Oficial [da] República Federativa do Brasil, Brasília, DF, n¹82, p. 19055-18694.

BARROS, M. E. D. Política de saúde: a complexa tarefa de enxergar a mudança onde tudo parece permanência. In: CANESQUI, A. M. (org.). Ciência Sociais e Saúde. São Paulo: HUCITEC-ABRASCO, 1997. cap. 6. p.113-133.

BLENDON, R. J. et al. Physician's views on quality of care: a five-country comparasion. Health Affairs, Milluood, v. 20, n. 6, p. 29, mar. 2001.

DONABEDIAN, A. La calidad de la atención médica: definición y métodos de evaluación. Mexico: La Prensa Médica Mexicana, 1980.

DONABEDIAN, A. The seven pillars of quality. Archives of Pathology and Laboratory Medicine, Northfield, v. 114, p. 115-1118, nov., 1990.

ESTEFANO, E.V.V. Satisfação dos recursos humanos no trabalho: um estudo de caso na biblioteca central da Universidade Federal de Santa Catarina. 1996. 117f. Dissertação (Mestrado em Engenharia) - Universidade Federal de Santa Catarina, Florianópolis, 1996.

FADEL, M. A. V.; FILHO, G. I. R. Percepção da qualidade em serviços públicos de saúde. In: ENCONTRO NACIONAL DE ENGENHARIA DE PRODUÇÃO, 26, 2006, Fortaleza. Anais...Fortaleza: Abepro, 2006. p. 681-693.

FITZSIMMONS, J. A.; FITZSIMMONS, M. J. Administração de serviços: operações, estratégia e tecnologia de informação. 4. ed. Porto Alegre: Bookman, 2005.

GEMMEL, P. et al. Patients' and personnel's perceptions of service quality and patient satisfaction in nuclear medicine. European Journal of Nuclear Medicine and Molecular Imaging, London, v. 29, n.9, p. 1109-17, 2002. 
GRONROOS, C. A. Marketing: gerenciamento e serviços. Rio de Janeiro: Elsevier, 2003.

HAIR, B.; B.; MONEY, A. H.; SAMOUEL, P. Fundamentos de métodos de pesquisa em administração. São Paulo: Bookman, 2005.

HARTZ, Z. M. A.; POUVOURVILLE, G. A avaliação da eficiência em saúde: a eficiência em questão Ciência \& Saúde Coletiva, Rio de Janeiro, v. 3, p. 68-82, 1998.

KARATEPE, O. M.; YAVA U.; BABAKUS E. Mensuring service quality of banks: scale development and validation. Journal of Retailing and Consumer Services, St. Louis, v. 12, n. 5, p. 373-838, 2005.

LOVELOCK, C.; WRIGHT, L. Serviços: marketing e gestão. São Paulo: Saraiva, 2004.

MEDINA, M. G.; AQUINO, R. Avaliando o Programa de saúde da família. In: SOUZA, M. F. (Org.) Os sinais vermelhos do PSF. São Paulo: Hucitec, 2002. cap. 8, p. 135151.

MEZOMO, J. C. Gestão da qualidade na saúde: princípios básicos. São Paulo: Manole, 2001.

NOGUEIRA-MARTINS, M. C. F. Humanização da assistência e formação do profissional de saúde. Psychiatry on line, Brazil, n. 8, maio, 2003.

PARASURAMAN, A.; ZEITHMAL, V. A.; BERRY, L. L.. Servqual: a multipleitem scale for measuring consumer perceptions of service quality. Journal of Retailing, St. Louis, v. 64, n. 1, p. 12-40, 1988.

PARENTE, J. Varejo no Brasil: gestão e estratégia. São Paulo: Atlas, 2000.

SOUZA JUNIOR, J. J. L. Qualidade: um enfoque por teoria da decisão. 2002. $106 f$. Dissertação (Mestrado em Engenharia de Produção) - Universidade Federal de Pernambuco, Recife, 2002

SOUZA, H. M. de. O futuro tecido no presente. Revista Brasileira de Saúde da Família, Brasília, DF, n. 1, p. 7-9, nov. 1999.

TANAKA, O. Y.; MELO, C. Reflexões sobre a avaliação em serviços de saúde e a adoção das abordagens qualitativa e quantitativa. In: BOSI, M. L. M.; MERCADO, F. J. Pesquisa qualitativa de serviços de saúde. Petrópolis: Vozes, 2004. p. 121136.

URDAN, A. T. A qualidade de serviços médicos na perspectiva do cliente. Revista de Administração de Empresas, São Paulo, v. 41, n. 4, p. 44-45, out./dez., 2001.

VUORI, H.. A qualidade da saúde. Divulgação em saúde para o debate, Londrina, 
n. 3, p. 17-24, 1991.

ZEITHMAL, V. A.; BITTER, M.J. Marketing de serviços: a empresa com foco no cliente. 2. ed. Porto Alegre: Bookman, 2003.

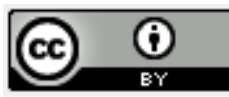

Artigo recebido em 28/07/2008 e aceito para publicação em 21/08/2010. 